\title{
Composite poly(DL-lactide-co-glycolide)/poly(acrylic acid) hydrogels synthesized using UV and gamma irradiation: comparison of material properties
}

\author{
Željko Janićijevića ${ }^{\mathrm{a} b}$, Ivica Vujčićc ${ }^{\mathrm{c}}$ Đorđe Veljovićd ${ }^{\mathrm{d}}$, Miloš Vujisića , Filip Radovanović $^{\mathrm{b}, *}$ \\ a University of Belgrade, School of Electrical Engineering, Bulevar Kralja Aleksandra 73, 11120, Belgrade, Serbia \\ ${ }^{\mathrm{b}}$ Institute of Technical Sciences of the Serbian Academy of Sciences and Arts, Knez Mihailova 35/IV, 11000, Belgrade, Serbia \\ ${ }^{\mathrm{c}}$ Vinča Institute of Nuclear Sciences, University of Belgrade, Mike Petrovića Alasa 12-14, 11351, Vinča, Belgrade, Serbia \\ ${ }^{\mathrm{d}}$ University of Belgrade, Faculty of Technology and Metallurgy, Karnegijeva 4, 11000, Belgrade, Serbia
}

\section{A R T I C L E I N F O}

\section{Keywords:}

PLGA

Composite hydrogel

Radiation-induced synthesis

Polymerization

Gamma irradiation

Ion exchange

\begin{abstract}
A B S T R A C T
Composite hydrogels capable of controlled drug delivery via ion exchange are an interesting group of materials for the construction of implantable drug reservoirs for electrically charged drugs. In this study, we synthesized composite poly(DL-lactide-co-glycolide)/poly(acrylic acid) (PLGA-PAA) hydrogels by sequential application of UV or gamma irradiation and traditional phase inversion. Physicochemical properties of the composite PLGAPAA hydrogels were investigated using Fourier transform infrared spectroscopy with attenuated total reflectance (FTIR-ATR), scanning electron microscopy (SEM), and differential scanning calorimetry (DSC). We examined the ion exchange capacity (IEC) and swelling behavior of these materials to determine their potential as drug reservoirs.

Composite PLGA-PAA hydrogel synthesized using UV irradiation (UV-PLGA-PAA) exhibited a porous microstructure with submicron-sized hydrogel-rich aggregates and homogeneous chemical composition. Swelling behavior and IEC of this material were highly reproducible. Composite PLGA-PAA hydrogels synthesized using gamma irradiation (G-PLGA-PAAs) had a less uniform microstructure with larger pores and micron-sized hydrogel-rich aggregates while exhibiting rather inhomogeneous chemical composition. These materials showed superior swelling properties, but a more variable IEC, compared to the material fabricated using UV irradiation. Results of DSC analysis showed a dose-dependent decrease in glass transition temperature for G-PLGA-PAAs indicating the effects of PLGA chain scission.

Our findings indicate that gamma irradiation is a possible alternative to UV irradiation in the synthesis of composite PLGA-PAA hydrogels which can modify or control important material properties. However, the synthesis protocol using gamma irradiation should be further optimized to improve the IEC reproducibility. In our future research, we will investigate the in vitro release of charged drugs from synthesized composite PLGAPAA hydrogels under physiological conditions.
\end{abstract}

\section{Introduction}

Biocompatible and biodegradable polymeric materials are important components of most implantable drug delivery systems. They are commonly employed in the form of drug reservoirs implanted or injected into the body which enable the storage and gradual release of drug molecules through various mechanisms (Kumar and Pillai, 2018).

Hydrogels have many beneficial properties for drug delivery which can be exploited to design efficient drug delivery systems (Li and Mooney, 2016). Some hydrogels can provide enhanced regulation of ion transport through electrostatic interactions, which makes them attractive for controlled release of charged drugs. However, most hydrogels typically suffer from poor mechanical properties due to spatial inhomogeneity (Okay, 2009) coupled with high swelling degrees in physiological environments. Hence, their use can be improved by introducing a matrix for mechanical support.

Composite materials comprising a biocompatible hydrophobic polymer matrix and a crosslinked hydrogel capable of ion exchange are interesting candidates for innovative drug reservoirs. Such materials can be used for efficient storage and controlled passive or active release

\footnotetext{
* Corresponding author.

E-mail addresses: filip.radovanovic@itn.sanu.ac.rs, filip@nanosys.ihtm.bg.ac.rs (F. Radovanović).
} 
of cationic drugs, as demonstrated by our previous studies (Janićijević et al., 2019; Janićijević and Radovanović, 2018).

Drug reservoirs must be sterilized before in vivo implantation to remove potential biological contamination. Various sterilization techniques can be used for this purpose with different degrees of success (Dai et al., 2016). Sterilization method of choice is defined by material properties and its intended application. One of the preferred and most potent sterilization methods for polymeric materials is gamma irradiation with the ${ }^{60} \mathrm{Co}$ source. The recommended sterilization dose for medical devices and pharmaceuticals is $25 \mathrm{kGy}$ (Silva Aquino, 2012). For radiation-sensitive polymers with low initial bioburden, it is beneficial to use lower sterilization doses. Adequacy of sterilization dose can be validated using various methods (Hasanain et al., 2014). Kim et al. have shown that polypyrrole biomaterials can be effectively sterilized with a minimum dose of $15 \mathrm{kGy}$ without significant alterations of cytocompatibility and electrical conductivity (Kim et al., 2018). For radiation-sensitive polymeric drug carriers, deterioration of relevant properties during sterilization may also be avoided by combining aseptic preparation and lower doses of high energy radiation, as proposed by Sintzel and coworkers (Sintzel et al., 1997).

Gamma irradiation is also well-known for its ability to induce polymerization of monomers in a solution without the use of additives, such as initiators and crosslinkers (Callinan, 1956). A major convenience of this technique is that the produced biomaterial is of high purity, as no initiator and related impurities remain in the matrix. Gamma irradiation can induce various effects, such as crosslinking and scission in polymers and networks, polymerization of pure and blended monomers, grafting onto synthetic and natural polymers, as well as chemical activation of organic materials by oxidation. Chain reactions leading to polymerization can be achieved with medium gamma-ray absorbed doses, while processes involving single steps or short kinetic chain length reactions typically require higher doses, as in the case of radiation crosslinking (Coqueret, 2008; Gupta and Anjum, 2003). Gamma rays could, therefore, be used for simultaneous synthesis, modification, and sterilization of polymeric materials or intermediate synthesis products.

In our previous research, we successfully synthesized composite implantable reservoirs comprising crosslinked poly(acrylic acid) (PAA) hydrogel and hydrophobic biocompatible polymer poly(DL-lactide-co- $\varepsilon$ caprolactone) (Janićijević et al., 2019). The synthesis protocol incorporated the UV irradiation of the initial solution followed by phase separation and solidification in the aqueous bath. Our subsequent preliminary tests suggested that the described approach with minor modifications can also be applied to the combinations of crosslinked PAA hydrogel with other hydrophobic polymer matrices.

Two issues arise in this synthesis process, but they can be circumvented by using gamma irradiation. First, synthesis with UV irradiation requires the use of photoinitiator (PI) bis(2,4,6-trimethylbenzoyl)-phenylphosphineoxide (Irgacure 819), a known skin sensitizer which is classified as hazardous under OSHA regulations (Irgacure 819, 2006). Second, the sterilization efficacy of UV irradiation is quite limited (Dai et al., 2016). Therefore, all steps in the synthesis of such implants must be conducted in aseptic conditions to avoid contamination, which increases the fabrication cost. The proposed solution is to replace UV irradiation with gamma irradiation in the first step of the synthesis and remove the PI from the initial solution.

PAA hydrogel is the reservoir component suitable for the storage of cationic drugs since it contains copious carboxyl groups allowing for ion exchange (Vuorio et al., 2003). It has been shown that gamma irradiation can induce the polymerization of acrylic acid to form PAA hydrogels with different sizes and properties (Abd El-Rehim et al., 2013; Sütekin and Güven, 2018). PAA microgels can also be synthesized using gamma irradiation of dilute aqueous solutions of PAA (Matusiak et al., 2018). Poly(DL-lactide-co-glycolide) (PLGA) is commonly used in drug delivery systems as a biodegradable and highly biocompatible polymer matrix (Makadia and Siegel, 2011). PLGA degrades via hydrolysis and enzymatic chain scission under physiological conditions, and its degradation products are then metabolized through the Krebs cycle (Kimura and Ogura, 2001). Influence of gamma irradiation on PLGA is well studied and documented (Carrascosa et al., 2003; Davison et al., 2018; Jo et al., 2012; Keles et al., 2015; Lee et al., 2003, 2002; Montanari et al., 1998). $N$-methyl-2-pyrrolidone (NMP) is a water-soluble organic solvent approved by the Food and Drug Administration and suitable for fast solidification of polymer implants (Jouyban et al., 2010). NMP was successfully used as a solvent for PLGA in the preparation of in situ forming polymeric systems using gamma irradiation of $25 \mathrm{kGy}$ (Rafienia et al., 2009, 2007). Quenching of PLGA solutions in NMP with aqueous solutions can be used to produce a porous hydrophobic matrix as demonstrated by previous studies of PLGA phase inversion dynamics (Brodbeck et al., 1999; Graham et al., 1999; Parent et al., 2013). The porosity of a PLGA polymer matrix within the composite drug reservoir could influence material properties relevant to drug delivery such as degradation rate, release kinetics, and release mechanism (Keles et al., 2014; Klose et al., 2006).

In this study, we synthesized implantable composite hydrogels comprising PLGA and crosslinked PAA using UV or gamma irradiation. Material properties of the obtained composite hydrogels were investigated and intercompared to preliminary estimate their applicability as reservoirs for cationic drugs.

\section{Experimental}

\subsection{Materials}

50:50 PLGA $\left(M_{\mathrm{w}}=(31.3-57.6) \mathrm{kDa}\right.$, ester-terminated) was supplied by DURECT Corporation. NMP (99\%), acrylic acid (AA) (99\%), and trimethylolpropane ethoxylate triacrylate (TMPTA) (average $M_{\mathrm{n}} \approx 912$ ) were purchased from Sigma-Aldrich. PI, Irgacure 819 , was kindly supplied by Ciba SC. Sodium hydroxide $(\mathrm{NaOH})$ (p.a., $>98 \%$ ) and potassium dihydrogen phosphate $\left(\mathrm{KH}_{2} \mathrm{PO}_{4}\right)$ (p.a., $\left.>99 \%\right)$ were obtained from Centrohem, Stara Pazova, Serbia. Hydrochloric acid ( $\mathrm{HCl})(37 \%)$ and $n$-heptane $(\geq 99 \%)$ were purchased from VWR Chemicals. Ethanol (p.a.) was obtained from Zorka Pharma, Šabac, Serbia.

An aqueous solution of $\mathrm{KH}_{2} \mathrm{PO} 4$ was titrated with the aqueous solution of $\mathrm{NaOH}$ to prepare the phosphate-buffered saline (PBS) of $\mathrm{pH}=7.4$ and ionic strength $I=0.154 \mathrm{M}$.

Chemicals were used as supplied, without additional purification. Aqueous solutions for our experiments were prepared using distilled water. Teflon rings (PTFE Flat Washer) had a nominal thickness of $1.575 \mathrm{~mm}$ and an internal diameter of $11.252 \mathrm{~mm}$. Fluorinated ethylene propylene non-stick film of $25 \mu \mathrm{m}$ thickness was obtained from Scientific Commodities, Inc.

\subsection{Synthesis of composite PLGA-PAA hydrogel using UV irradiation}

The composite hydrogel was prepared using the modified liquid phase inversion process initially devised for membranes (Mulder, 1996), similarly as described in our previous study (Janićijević et al., 2019). AA monomer and trifunctional crosslinker TMPTA were added to the polymer base (PLGA) solution and copolymerized before the immersion in PBS, which completed phase separation and solidification of the composite hydrogel.

For the synthesis experiments, $30 \mathrm{wt} \%$ solution of PLGA in NMP was prepared by stirring overnight, then combined with the solution of photopolymerizable components (AA, TMPTA, and PI) in NMP by stirring in an amber vial cooled within an ice bath. The solution obtained after mixing was transparent, viscous, and homogeneous. The composition of the initial solution is given as $16 \mathrm{wt} \%$ of PLGA, $3.41 \mathrm{mmol} / \mathrm{g}$ of AA, and $10 \mathrm{~mol} \%$ of TMPTA. The concentration of the PLGA polymer base is expressed in wt $\%$ of the initial solution mass. AA concentration is given in $\mathrm{mmol} / \mathrm{g}$ of the final dry sample assuming the 
$100 \%$ yield of reactant conversion. Crosslinker concentration is expressed as mol\% with respect to the AA concentration. A predetermined amount of the initial solution was placed within the mold comprising the Teflon ring sealed at the top and bottom with the transparent nonstick film. The solution in the mold was subjected to UV irradiation of the wavelength $\lambda=365 \mathrm{~nm}$ through the glass plate for $3 \mathrm{~min}$ to achieve the irradiation dose of $3.6 \mathrm{~J} / \mathrm{cm}^{2}$, as measured by the YK-35UV light meter. Exposure to UV irradiation initiated the processes of AA polymerization and crosslinking, which resulted in gel formation. After UV curing, the gel was dropped into the PBS solution using a non-stick plunger to finalize phase separation and solidification, which resulted in the formation of the hydrogel-filled white disk (see Fig. S1 in the Supplementary material). After the synthesis, composite PLGA-PAA hydrogel disks were stored for $24 \mathrm{~h}$ in the aqueous bath containing an excessive amount of fresh PBS solution to extract unreacted components. After this period, the composite PLGA-PAA hydrogel disks were either dried or used immediately in the wet state to perform the experiments.

Composite PLGA-PAA hydrogel synthesized using UV irradiation is designated in the further text as UV-PLGA-PAA.

\subsection{Synthesis of composite PLGA-PAA hydrogels using gamma irradiation}

An initial solution for the synthesis using gamma irradiation was prepared using the same formulation as for the synthesis with UV irradiation, but without the PI as a component. Molds filled with the initial precursor solution and sealed with the transparent non-stick film were packed into polypropylene ziplock bags and placed horizontally at the predefined positions in front of the source of gamma radiation.

Irradiation was performed with a ${ }^{60} \mathrm{Co}$ gamma-source at the Radiation Unit for Industrial Sterilization and Conservation, Vinca Institute of Nuclear Sciences, at room temperature, in contact with air. Radiation Unit has operated since 1978, and the services are mainly provided for industry (sterilization of medical products, irradiation of food), and scientific research. The current activity of ${ }^{60} \mathrm{Co}$ gammasource is around $100 \mathrm{kCi}$. Samples were treated with three different doses of gamma radiation: $17 \mathrm{kGy}, 25 \mathrm{kGy}$, and $33 \mathrm{kGy}$. The dose rate was $8.1 \mathrm{kGy} / \mathrm{h}$ for doses of $17 \mathrm{kGy}$ and $33 \mathrm{kGy}$, while three different dose rates were used for the samples irradiated with a dose of $25 \mathrm{kGy}$ : $6.1 \mathrm{kGy} / \mathrm{h}, 8.1 \mathrm{kGy} / \mathrm{h}$, and $10.1 \mathrm{kGy} / \mathrm{h}$. Dosimetry measurements for gamma-ray field mapping were performed by the ethanol-chlorobenzene solution (ECB) dosimetry ("ISO/ASTM 51538:2017 - Practice for use of the ethanol-chlorobenzene dosimetry system," 2017). The ECB solution is a well-known routine standard dosimeter for evaluating the absorbed dose of high-energy radiation (Kovács et al., 1987; Razem and Dvornik, 1972). Measurements were made by oscillotitrator OK302/1 supplied by Radeliks Electrochemical Instruments, Budapest, Hungary. Calibration is performed using EBC dosimeters irradiated at High Dose Reference Laboratory of Risø National Laboratory, Denmark.

In this synthesis, gamma irradiation initiated the processes of polymerization and crosslinking of AA leading to the formation of gels with a cloudy appearance. After gamma irradiation, gels were removed from the molds using the nonstick plunger, dropped into the PBS solution and further treated in the same manner as the composite hydrogel fabricated using UV irradiation. The final products of the synthesis were hydrogel-filled disks of pale yellowish color (see Fig. S2 in the Supplementary material).

Composite PLGA-PAA hydrogels synthesized using gamma irradiation are designated in the further text as G-PLGA-PAAs.

\subsection{Materials characterization}

Composite PLGA-PAA hydrogels were characterized using the techniques of Fourier transform infrared spectroscopy with attenuated total reflectance (FTIR-ATR), Scanning electron microscopy (SEM), and Differential scanning calorimetry (DSC).
FTIR-ATR analysis of chemical composition was conducted by Thermo Scientific Nicolet iS10 FTIR Spectrometer with Smart iTX ATR Diamond accessory. All spectra were acquired in the range of $400-4000 \mathrm{~cm}^{-1}$ (resolution of $0.5 \mathrm{~cm}^{-1}$ ). Obtained raw spectra were then normalized to the highest peak intensity in absorbance mode for qualitative presentation.

The microstructure of fractured surfaces along the cross section of composite hydrogels was investigated using the field emission scanning electron microscope (FE-SEM) (TESCAN MIRA 3 XMU) operated at $20 \mathrm{keV}$. Before the recording, dry samples were cooled in liquid nitrogen, fractured, and sputtered with a thin layer of gold. Analysis of particle size using the measurement of Feret diameter was performed with the ImageJ 1.52j image processing program (Schneider et al., 2012).

DSC characterization of glass transitions in composite hydrogels was performed using the instrument SETARAM DSC 131 Evo programmed and controlled by CALISTO software. Samples were placed in hermetically sealed aluminum pans of $30 \mu \mathrm{l}$ volume, and the recordings were carried out in the temperature range from 10 to $140^{\circ} \mathrm{C}$ with the heating rate of $10^{\circ} \mathrm{C} / \mathrm{min}$ under nitrogen purging. DSC thermograms were normalized to the unit mass of PLGA in the analyzed sample according to its mass fraction. Glass transition temperature $\left(\mathrm{T}_{\mathrm{g}}\right)$ was determined by using the halfwidth of the enthalpic relaxation peak associated with the glass transition.

Protocol of drying by solvent exchange was utilized to prepare dry samples of composite PLGA-PAA hydrogels for FTIR-ATR, SEM and DSC examinations. Wet as-synthesized samples with absorbed PBS were soaked for $1 \mathrm{~h}$ in ethanol and then transferred for $1 \mathrm{~h}$ in heptane. The samples were then left to dry in air at ambient conditions for at least $72 \mathrm{~h}$.

\subsection{Swelling degree measurements}

Samples were prepared for mass swelling degree (MSD) measurements by equilibration in PBS for $24 \mathrm{~h}$. Initially, the mass of the wet sample $m_{\mathrm{w}}$ was measured. The sample was then dried in an oven at $100{ }^{\circ} \mathrm{C}$ for $2 \mathrm{~h}$ under atmospheric pressure. Finally, the mass of the dried sample $m_{\mathrm{d}}$ was measured. The experiment was carried out in duplicate.

MSD was determined based on the wet sample mass, $m_{\mathrm{w}}$, and the dry sample mass, $m_{\mathrm{d}}$, using the following formula

$M S D=\frac{\left(m_{w}-m_{d}\right)}{m_{d}} \cdot 100(\%)$

\subsection{Swelling kinetics measurements}

Kinetics of composite hydrogel swelling in PBS was recorded by measuring the MSD at predefined time intervals until the MSD value approached equilibrium. At first, the sample was dried in air at ambient temperature and pressure for $72 \mathrm{~h}$. Mass of dry sample was determined before the experiment. Experiments were initiated by immersing the sample in an excess amount of PBS within a Petri dish. At predefined times, the sample was taken out of PBS and carefully blotted with filter paper. The wet mass of the sample was recorded, and the sample was quickly returned to the PBS solution. This procedure was repeated several times throughout $60 \mathrm{~min}$. Swelling kinetics experiments were carried out in triplicate.

\subsection{Measurements of carboxyl group concentration (ion exchange capacity)}

Carboxyl group concentration was measured by using the conductometric acid-base titration method. One-quarter of the dry diskshaped sample (about $15 \mathrm{mg}$ ) was cut into smaller pieces and placed in $100 \mathrm{ml}$ of $0.1 \mathrm{M} \mathrm{HCl}$ solution. Carboxyl groups in the sample were protonated by mild stirring of the sample in the solution for $1 \mathrm{~h}$. The 
sample was then taken out from the solution, rinsed with an excess amount of distilled water and blotted with tissue paper. The protonated sample was immersed in $40 \mathrm{ml}$ of $0.01 \mathrm{M} \mathrm{NaOH}$ solution and stirred for $1 \mathrm{~h}$ in a closed beaker. The sample was then removed from the remaining solution, thoroughly rinsed with distilled water, and dried for $2 \mathrm{~h}$ at $100^{\circ} \mathrm{C}$ in the oven under atmospheric pressure. Finally, the dry sample was weighed.

Two $15 \mathrm{ml}$ aliquots of residual $\mathrm{NaOH}$ solution were titrated with $0.01 \mathrm{M} \mathrm{HCl}$, and two $15 \mathrm{ml}$ aliquots of $0.01 \mathrm{M} \mathrm{NaOH}$ solution were titrated with $0.01 \mathrm{M} \mathrm{HCl}$ as blank probes. The conductometric titration curves were recorded with a conductometer, and the equivalence point was determined. The experiment was carried out in duplicate.

The concentration of carboxyl groups $\left(\mathrm{C}_{\mathrm{cg}}\right)$ was obtained using the formula:

$C_{c g}(\mathrm{mmol} / \mathrm{g})=0.4\left(1-V_{2} / V_{1}\right) / m_{d}$

where $V_{1}$ is the $0.01 \mathrm{M} \mathrm{HCl}$ solution volume consumed for blank probe titration, $V_{2}$ the $0.01 \mathrm{M} \mathrm{HCl}$ solution volume consumed for the titration of residual solution aliquot, and $m_{\mathrm{d}}$ dry sample mass.

\subsection{Statistical analysis}

Presented quantitative data are reported as the mean \pm standard deviation (SD) of the designated sample. Mean value and SD are calculated based on the stated number of measurements. Statistical significance between sample groups synthesized using different gamma irradiation parameters was determined by one-way analysis of variance (one-way ANOVA); $p$-values smaller than 0.05 were considered as statistically significant. The assumption of homoscedasticity was checked with the Bartlett's or Levene's test; $p$-value of 0.05 was taken as the significance level.

\section{Results and discussion}

\subsection{FTIR-ATR characterization}

FTIR-ATR spectra of pure PLGA and UV-PLGA-PAA (in the wet and dry states) are shown in Fig. 1. Representative FTIR-ATR spectra of GPLGA-PAAs (in the wet and dry states) are presented in Fig. 2. Band assignments in the infrared spectra of PLGA were studied in detail and summarized by Vey and coworkers (Vey et al., 2011). Assignments for vibration bands in the infrared spectra of pure PAA in the wet and dry state were thoroughly discussed by Todica et al. (2015).

The spectrum of pure PLGA illustrates the hydrophobic nature of the polymer and contains multiple vibration bands which indicate the presence of lactic and glycolic units. Vibration bands at $2947 \mathrm{~cm}^{-1}$ and $2996 \mathrm{~cm}^{-1}$ are assigned to the symmetric and anti-symmetric stretching of $\mathrm{CH}_{3}$, while the bands at 2853 and $2925 \mathrm{~cm}^{-1}$ are assigned to the symmetric and anti-symmetric stretching of $\mathrm{CH}_{2}$, respectively. The strong peak at $1746 \mathrm{~cm}^{-1}$ is assigned to the $\mathrm{C}=\mathrm{O}$ stretching vibration. Bands at 1364 and $1383 \mathrm{~cm}^{-1}$ correspond to the symmetric bending, while the band at $1451 \mathrm{~cm}^{-1}$ corresponds to the anti-symmetric bending of $\mathrm{CH}_{3}$ in lactic units. Vibration bands at 1394 and $1422 \mathrm{~cm}^{-1}$ represent the wagging and bending modes of $\mathrm{CH}_{2}$ in glycolic units, respectively. The band at $1269 \mathrm{~cm}^{-1}$ is assigned to the $\mathrm{CH}_{2}$ twisting vibration. Peaks at 1084 and $1164 \mathrm{~cm}^{-1}$ correspond to the symmetric and anti-symmetric stretching COC vibrations, respectively. Vibration bands at 958,1050 , and $1130 \mathrm{~cm}^{-1}$ are assigned to the rocking of $\mathrm{CH}_{3}, \mathrm{C}-\mathrm{CH}_{3}$ stretching, and anti-symmetric rocking of $\mathrm{CH}_{3}$ in lactic units, respectively.

The spectrum of dry UV-PLGA-PAA contains additional peaks at $1352 \mathrm{~cm}^{-1}$ corresponding to $\mathrm{C}-\mathrm{H}$ rocking and $1570 \mathrm{~cm}^{-1}$ assigned to $\mathrm{C}-\mathrm{O}$ stretching of carboxyl groups. Minor changes in band shapes are visible in the $1500-1300 \mathrm{~cm}^{-1}$ and $3000-2800 \mathrm{~cm}^{-1}$ regions due to the presence of crosslinked PAA backbone. The bands in the 1500$1300 \mathrm{~cm}^{-1}$ region are also overlapped with other $\mathrm{C}-\mathrm{O}$ stretching bands of PAA.

Wet UV-PLGA-PAA exhibits few additional peaks in the spectrum mainly confirming the presence of PAA hydrogel formation. The peak at $1636 \mathrm{~cm}^{-1}$ arises from the hydrogen bonding of carboxyl groups forming dimers and oligomers, while the peak at $3357 \mathrm{~cm}^{-1}$ is assigned to the symmetric $\mathrm{H}-\mathrm{O}-\mathrm{H}$ stretching of hydrogen-bonded water. The weak band corresponding to $\mathrm{C}-\mathrm{H}$ stretching at $2881 \mathrm{~cm}^{-1}$ also appears in the spectrum. Hydrogen bonding in the wet UV-PLGA-PAA causes the slight shifts of band positions which are more pronounced for the bands associated with carboxyl and carbonyl groups.

Infrared spectra of UV-PLGA-PAA acquired on different locations within the sample indicate uniform chemical composition, as shown in Fig. S3 in the Supplementary material. Conversely, G-PLGA-PAAs appear to be spatially inhomogeneous regarding chemical composition for all sets of applied irradiation parameters, as illustrated in Figs. S4-S8 in the Supplementary material. However, representative features of FTIR-ATR spectra can still be identified for the G-PLGA-PAAs (see Fig. 2). These features in the examined composite hydrogels depend on the specific irradiation parameters, but most of them can also appear locally in all gamma irradiated samples due to the stochastic (i.e., spatially discrete) nature of gamma-ray interactions.

Spectra of G-PLGA-PAAs synthesized using the doses of 17 and $25 \mathrm{kGy}$ generally exhibit similar bands as for the UV-PLGA-PAA. Some subtle pattern differences are observed in the spectral $1500-1300 \mathrm{~cm}^{-1}$ and $3000-2800 \mathrm{~cm}^{-1}$ regions mainly corresponding to the deformation and stretching vibrations of $\mathrm{CH}_{2}$ and $\mathrm{CH}_{3}$. Pattern differences corresponding to the regions of deformation and stretching vibrations of $\mathrm{CH}_{2}$ and $\mathrm{CH}_{3}$ are more obvious in the case of G-PLGA-PAAs synthesized

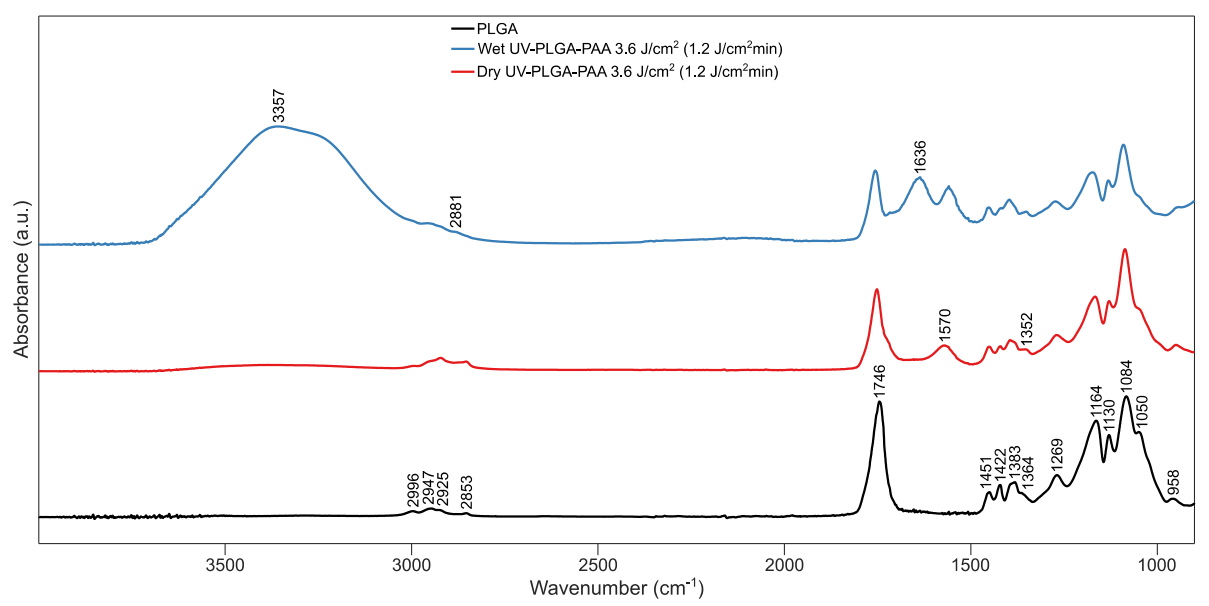

Fig. 1. Normalized FTIR-ATR spectra of pure PLGA and UV-PLGA-PAA (dose $3.6 \mathrm{~J} / \mathrm{cm}^{2}$; dose rate $1.2 \mathrm{~J} / \mathrm{cm}^{2} \mathrm{~min}$ ). 

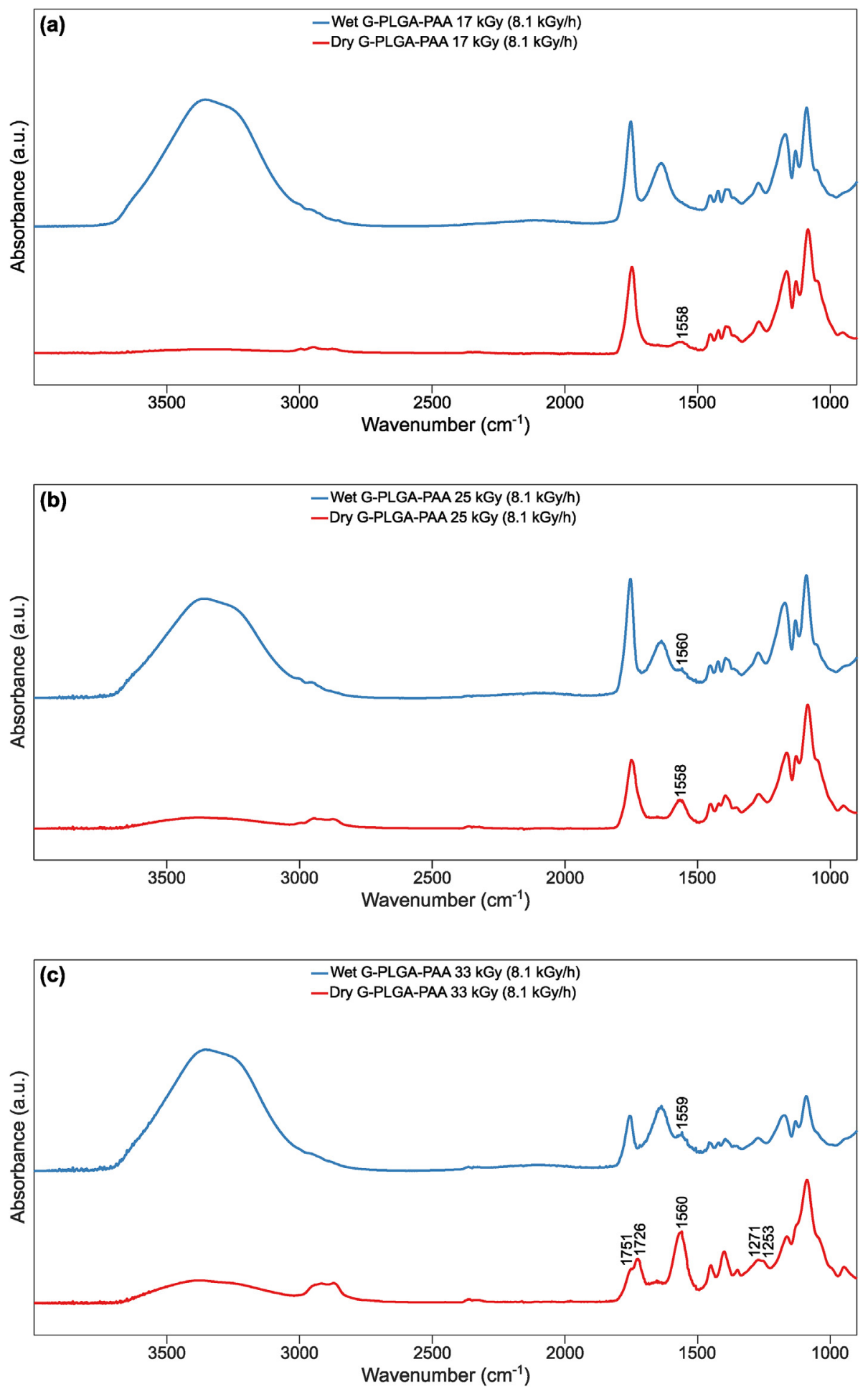

Fig. 2. Normalized representative FTIR-ATR spectra of G-PLGA-PAAs synthesized using different irradiation parameters (dose (kGy)/dose rate (kGy/h)): 17/8.1 (a), 25/8.1 (b), and 33/8.1 (c).

using the dose of $33 \mathrm{kGy}$.

Minor changes in chemical bonding and composition are hard to detect and quantify from FTIR-ATR spectra of polyesters such as PLGA even for much higher applied doses of gamma irradiation (Jo et al., 2012). Lack of systematic changes within the mentioned regions in FTIR-ATR spectra can also be ascribed to the random nature of chain scission process (Oliveira et al., 2012) that can occur during gamma irradiation of PLGA.

The FTIR-ATR spectrum of G-PLGA-PAA synthesized using the dose of $33 \mathrm{kGy}$ exhibits additional changes such as peak splitting (see Fig. 2c). Peak splitting can be observed in the dry state as two strongly overlapping bands corresponding to the $\mathrm{C}=\mathrm{O}$ stretching (at 1726 and $1751 \mathrm{~cm}^{-1}$ ) and $\mathrm{CH}_{2}$ twisting (at 1253 and $1271 \mathrm{~cm}^{-1}$ ) vibrations. Such changes in $\mathrm{C}-\mathrm{H}$ and $\mathrm{C}=\mathrm{O}$ bonds could be explained by the concept of cage effect. The cage effect occurs when initially formed radicals stay trapped and recombine locally before diffusing out of the active cage (Chu and Campbell, 1982; Loo et al., 2005).

Intensity ratios of the $\mathrm{C}=\mathrm{O}$ stretching peak and major $\mathrm{C}-\mathrm{O}$ 
stretching peak of crosslinked PAA (at around $1560 \mathrm{~cm}^{-1}$ ) also vary for the dry state in all recorded FTIR-ATR spectra of G-PLGA-PAAs. This variation can be attributed to the formation of more branched structures of PAA hydrogel (caused by the competition between inter- and intra-molecular crosslinking (Ghobashy, 2018) as well as random chain scission) and the possible increase in carboxyl group content of the composite hydrogels due to gamma irradiation of PLGA (Yoshioka et al., 1995).

The major C-O stretching peak of crosslinked PAA in the FTIR-ATR spectrum of wet UV-PLGA-PAA exhibits increased intensity and shifts towards lower wavenumbers which indicates the participation of $\mathrm{C}-\mathrm{O}$ groups in hydrogen bonding of carboxyl groups. The intensity of this peak in FTIR-ATR spectra of wet G-PLGA-PAAs is much weaker, or the peak becomes completely overlapped by the peak at $1636 \mathrm{~cm}^{-1}$, while there is no shift in peak position. Such peak properties indicate significantly reduced involvement of $\mathrm{C}-\mathrm{O}$ groups in hydrogen bonding. Reduced engagement of $\mathrm{C}-\mathrm{O}$ groups is possible in PAA hydrogels with a more branched structure where water molecules primarily become attached to terminal chain carboxyl groups via hydrogen bonding with $\mathrm{C}=\mathrm{O}$ group (Todica et al., 2015).

It should be noted that the dose rate parameter variation in the investigated range did not produce any significant systematic effects detected within the FTIR-ATR spectra of G-PLGA-PAAs.

\subsection{DSC characterization}

DSC thermograms of pure PLGA and synthesized composite PLGAPAA hydrogels are shown in Fig. 3, and the calculated values of $\mathrm{T}_{\mathrm{g}}$ are listed in Table 1. Detected glass transitions of PLGA are overlapped with the enthalpic relaxation peaks commonly caused by mechanical stresses imposed during the fabrication process or prolonged storage under $\mathrm{T}_{\mathrm{g}}$ (Gabbott, 2008). Two types of $\mathrm{T}_{\mathrm{g}}$ shifts can be observed in our samples. $\mathrm{T}_{\mathrm{g}}$ of UV-PLGA-PAA is shifted higher by almost $4{ }^{\circ} \mathrm{C}$ compared to pure PLGA. The $\mathrm{T}_{\mathrm{g}}$ shift may be ascribed to the incomplete phase separation between PLGA and PAA resulting in entangled polymer chains, but also to the increase in mean molecular weight. Quenching of irradiated composite hydrogels in PBS solution causes precipitation of PLGA chains. PLGA chains of higher molecular weight have a stronger tendency to precipitate and solidify in the nonsolvent, while smaller oligomers may diffuse out together with NMP molecules. The net result would be an increase in the molecular weight of PLGA, which could explain the observed increase in $\mathrm{T}_{\mathrm{g}}$.

Described causes of $\mathrm{T}_{\mathrm{g}}$ increase are countered by the process of main chain scission in G-PLGA-PAA samples. The process of chain scission increases the free volume and allows for more intense chain motion. Additional movement of polymer chains results in a lower $\mathrm{T}_{g}$ representing the temperature required to diminish chain motion and transform the polymer into a glassy state (Davison et al., 2018). Chain scission dominates in the investigated dose range for PLGA as confirmed by previous studies and leads to the dose-dependent $\mathrm{T}_{\mathrm{g}}$ decrease (Davison et al., 2018; Jo et al., 2012). As a result of these opposing effects, $T_{g}$ of PLGA in samples irradiated with the lowest dose of $17 \mathrm{kGy}$ has a value between those for pure PLGA and UV-PLGA-PAA samples. The value of $\mathrm{T}_{\mathrm{g}}$ gradually decreases with the irradiation dose to reach its minimum at the dose of $33 \mathrm{kGy}$. As $\mathrm{T}_{\mathrm{g}}$ declines and approaches body temperature, degradation time of the implanted material may shorten due to the premature loss of structural integrity (Yoshioka et al., 1995).

Influence of dose rate appears to be minor in the investigated range since all $\mathrm{T}_{\mathrm{g}}$ values differ less than $1.6^{\circ} \mathrm{C}$. Although the findings are not conclusive due to limited measurement accuracy, they may indicate a weak trend of $\mathrm{T}_{\mathrm{g}}$ increase for a greater dose rate.

Increase in gamma irradiation dose leads to the reduction in intensity of enthalpic relaxation peaks detected in the corresponding DSC thermograms. The enthalpic relaxation peak almost disappears in the sample irradiated with $33 \mathrm{kGy}$. Hence, chain scission also reduces residual mechanical stresses which arise as a consequence of the synthesis
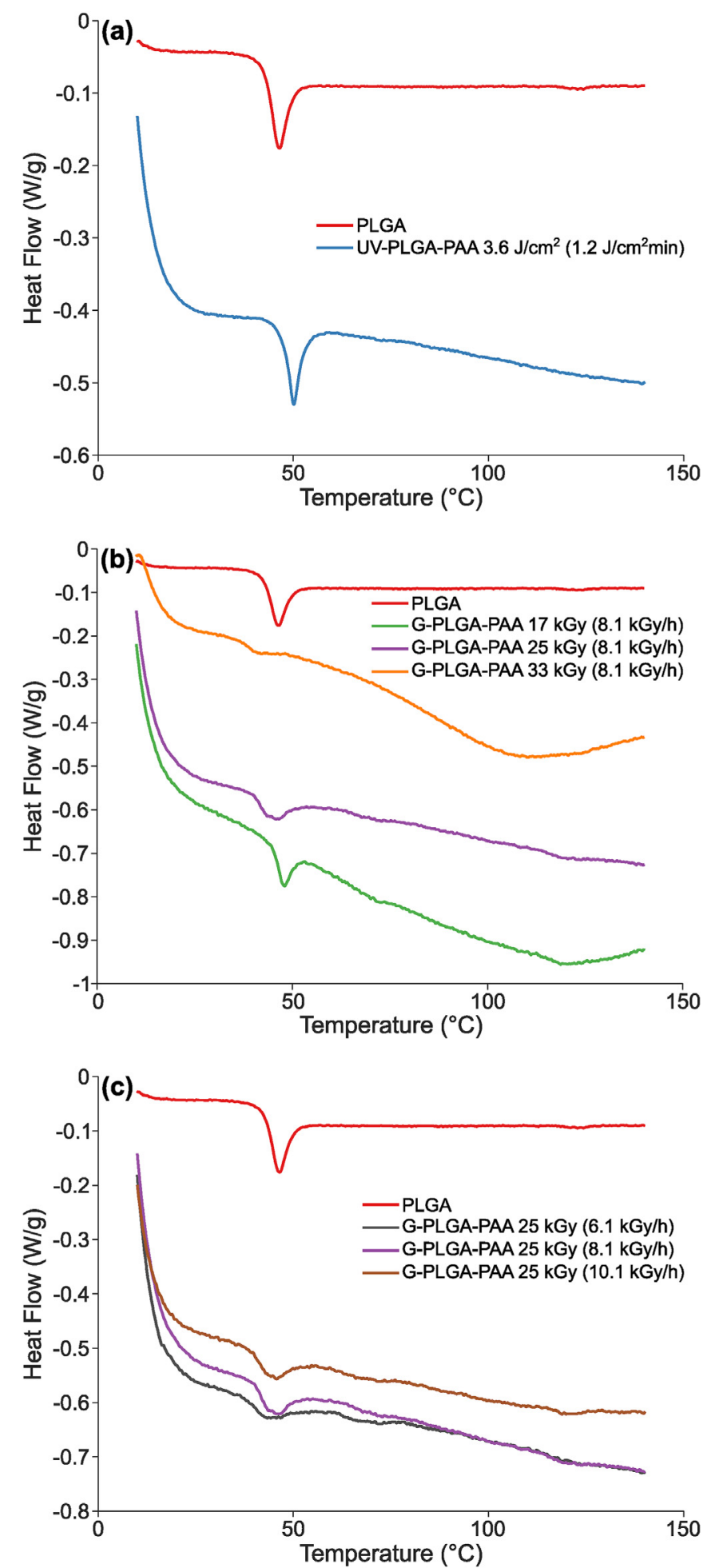

Fig. 3. DSC thermograms of composite PLGA-PAA hydrogels: (a) UV-PLGA-PAA (dose $3.6 \mathrm{~J} / \mathrm{cm}^{2}$; dose rate $1.2 \mathrm{~J} / \mathrm{cm}^{2} \mathrm{~min}$ ), (b) G-PLGA-PAAs synthesized using the applied doses of 17, 25, and $33 \mathrm{kGy}$ (with the constant dose rate of $8.1 \mathrm{kGy} /$ h), and (c) G-PLGA-PAAs synthesized using the applied dose rates of 6.1, 8.1, and $10.1 \mathrm{kGy} / \mathrm{h}$ (with the constant dose of $25 \mathrm{kGy}$ ). DSC thermogram of pure PLGA is shown as a reference.

process.

The obtained results for $\mathrm{T}_{\mathrm{g}}$ of PLGA may be affected by the moisture content of the composite PLGA-PAA hydrogels after drying by solvent exchange. Residual water could act as a plasticizer and lower the measured value of $\mathrm{T}_{\mathrm{g}}$. According to the findings of additional thermal 
Table 1

Glass transition temperature $\left(\mathrm{T}_{\mathrm{g}}\right)$ of composite PLGA-PAA hydrogels synthesized under different conditions.

\begin{tabular}{llc}
\hline Sample type & Irradiation parameters (dose, dose rate) & $\mathbf{T}_{\mathbf{g}}\left({ }^{\circ} \mathbf{C}\right)$ \\
\hline PLGA & None & 46.18 \\
UV-PLGA-PAA & $3.6 \mathrm{~J} / \mathrm{cm}^{2}, 1.2 \mathrm{~J} / \mathrm{cm}^{2} \mathrm{~min}$ & 50.11 \\
G-PLGA-PAA & $17 \mathrm{kGy}, 8.1 \mathrm{kGy} / \mathrm{h}$ & 48.18 \\
G-PLGA-PAA & $25 \mathrm{kGy}, 6.1 \mathrm{kGy} / \mathrm{h}$ & 44.42 \\
G-PLGA-PAA & $25 \mathrm{kGy}, 8.1 \mathrm{kGy} / \mathrm{h}$ & 45.30 \\
G-PLGA-PAA & $25 \mathrm{kGy}, 10.1 \mathrm{kGy} / \mathrm{h}$ & 46.00 \\
G-PLGA-PAA & $33 \mathrm{kGy}, 8.1 \mathrm{kGy} / \mathrm{h}$ & 42.32 \\
\hline
\end{tabular}

analysis, the moisture content is similar between samples and only accounts for several percent of the sample mass (for details refer to the section Estimation of moisture content in solvent exchange dried composite hydrogels in the Supplementary material).

In order to assess the detectability of $\mathrm{T}_{g}$ for PAA in the composite PLGA-PAA hydrogels and interpret their DSC thermograms above $60^{\circ} \mathrm{C}$, we also carried out additional DSC characterization. Our findings indicate that minor endothermic events and endothermic peaks appearing above $60^{\circ} \mathrm{C}$ mainly correspond to the elimination of absorbed moisture and bound water (for details refer to the section Analysis of the poly (acrylic acid) glass transition within composite hydrogels in the Supplementary material).

\subsection{SEM characterization}

SEM images of cross sections shown in Fig. 4 illustrate the microstructure of composite PLGA-PAA hydrogels. All samples exhibit a heterogeneous microstructure comprising dispersed PAA-rich spheroidal particles attached to the porous PLGA-rich matrix. Such microstructure results from the complex interplay of several phenomena during the synthesis. Irradiation initiates the crosslinking of AA and the process of phase separation. UV irradiation is uniformly absorbed due to the presence of a dissolved PI, and it leads to the formation of crosslinked PAA. Trifunctional crosslinker TMPTA polymerizes faster compared to the monofunctional AA monomers, and after crosslinker depletion, hydrogel-rich regions grow to form many small particle-like aggregates.

${ }^{60} \mathrm{Co}$ gamma rays have sufficient energy (on average $1.25 \mathrm{MeV}$ per photon) to form free radicals from AA, TMPTA, and PLGA molecules. They interact at random locations within the initial solution volume and can lead to various interactions with different probabilities, such as chain scission and crosslinking of intermolecular or intramolecular nature (Ferry et al., 2016). Gamma-ray-induced polymerization rate is commonly significantly lower than the UV induced polymerization rate due to the less efficient and non-selective radical formation. Slow polymerization and stochastic nature of material interactions with gamma rays allow for more significant contributions of free radical propagation and diffusion. The onset of AA polymerization occurs in randomly distributed centers in the initial solution volume. Polymerization then proceeds in the vicinity of these centers mainly via diffusion and propagation of free radicals. As in the case of UV irradiation, polymerization proceeds more rapidly for the trifunctional crosslinker TMPTA compared to the monofunctional AA monomers. Hence, a reduced number of larger particle-like PAA-rich aggregates is formed in comparison with UV-PLGA-PAA. Completion of phase separation and solidification for both composite hydrogel types take place under unstable thermodynamic conditions in the PBS solution, which acts as nonsolvent during phase inversion.

The porous structure of the PLGA support can be explained by the specific phase inversion dynamics of PLGA solutions in NMP when quenched with aqueous solutions, as shown in previous studies (Brodbeck et al., 1999; Graham et al., 1999). The high rate of solvent diffusion to the surrounding aqueous medium leads to the rapid precipitation of PLGA. As a result of this process, the solidified porous polymer matrix is formed.

Hydrophilic PAA-rich aggregates remain attached to the hydrophobic PLGA support due to the residual common chains. Such chains result from incomplete polymer/polymer demixing during phase separation as was also the case with the porous hydrophobic polyethersulfone matrix and PAA in our previous study (Janićijević and Radovanović, 2018).

The overall morphology of the composite PLGA-PAA hydrogels is similar, but the clear difference can be observed between the UV-PLGAPAA and G-PLGA-PAA samples. Size of PAA-rich aggregates in UVPLGA-PAA samples is $(0.62 \pm 0.10) \mu \mathrm{m}(\mathrm{n}=40$ particles), while the size of the PAA-rich aggregates in G-PLGA-PAA samples is (1.24 \pm 0.17$) \mu \mathrm{m}$ (pooled statistics of $n=200$ particles). The larger size of PAA-rich aggregates in G-PLGA-PAA samples compared to those in UV-PLGA-PAA samples may be a result of slower kinetics, which would allow more time for the aggregate growth before depletion of monomers. However, more research is needed to validate this hypothesis. Spatial nonuniformity of microstructural features is more pronounced in G-PLGA-PAAs compared to UV-PLGA-PAA. SEM analysis indicates that G-PLGA-PAAs synthesized with the dose of $25 \mathrm{kGy}$ and the dose rate of $6.1 \mathrm{kGy} / \mathrm{h}$ exhibit an even higher degree of microstructural nonuniformity and greater porosity compared to other GPLGA-PAAs. This finding can potentially be explained by the lowest dose rate which allows sufficient time for more complex interactions of free radicals leading to greater structural and compositional inhomogeneity.

\subsection{Ion exchange capacity}

As determined from the measurements of $\mathrm{C}_{\mathrm{cg}}$, ion exchange capacity (IEC) of all samples is approximately $2 \mathrm{mmol} / \mathrm{g}$ of the dry mass (see Fig. 5). This value is smaller than IEC of commercial pharmaceutical grade ion exchange resins, such as Smopex $^{\circledR}-102(6.4 \mathrm{mmol} / \mathrm{g})$ (Malinovskaja et al., 2013), but similar to the values obtained in our previous study (Janićijević et al., 2019). IEC of UV-PLGA-PAA is more reproducible and exhibits only a slight variation among samples. On the other hand, the IEC of G-PLGA-PAAs is highly variable. Statistical analysis showed no significant difference in IEC among G-PLGA-PAA samples synthesized with different irradiation parameters. The variability can be attributed to the inhomogeneous microstructure (see Fig. 4c-l) of the samples and macroscopic inhomogeneities in chemical composition (see Figs. S4-S8 in the Supplementary material).

\subsection{Swelling degree}

MSD of composite PLGA-PAA hydrogels is presented in Fig. 6. Results suggest that UV-PLGA-PAA samples exhibit about 14\% lower MSD compared to that of G-PLGA-PAA samples. All G-PLGA-PAAs show similar MSDs, which are practically independent of the irradiation parameters in the investigated range.

The differences in MSD can be ascribed to the different microstructure, the spatial structure of the PAA hydrogel network, and hydrophilicity of UV-PLGA-PAA and G-PLGA-PAAs. G-PLGA-PAA samples show inhomogeneous microstructure with larger pores which allows easier penetration of the aqueous electrolyte. Due to the crosslinking mechanisms characteristic for gamma irradiation, hydrogel with the more branched network is formed, which exposes more hydrophilic groups on its surface. Gamma irradiation in the dose range up to $25 \mathrm{kGy}$ could also lead to the increased content of polar carboxyl groups in PLGA (Yoshioka et al., 1995), which increases the overall hydrophilicity of the composite PLGA-PAA hydrogel.

Swelling degree of PAA hydrogel in all composite PLGA-PAA hydrogels is limited by the PLGA matrix which provides mechanical support. Similar MSDs for all gamma irradiated samples indicate that the mechanical integrity of the PLGA matrix is not significantly affected 


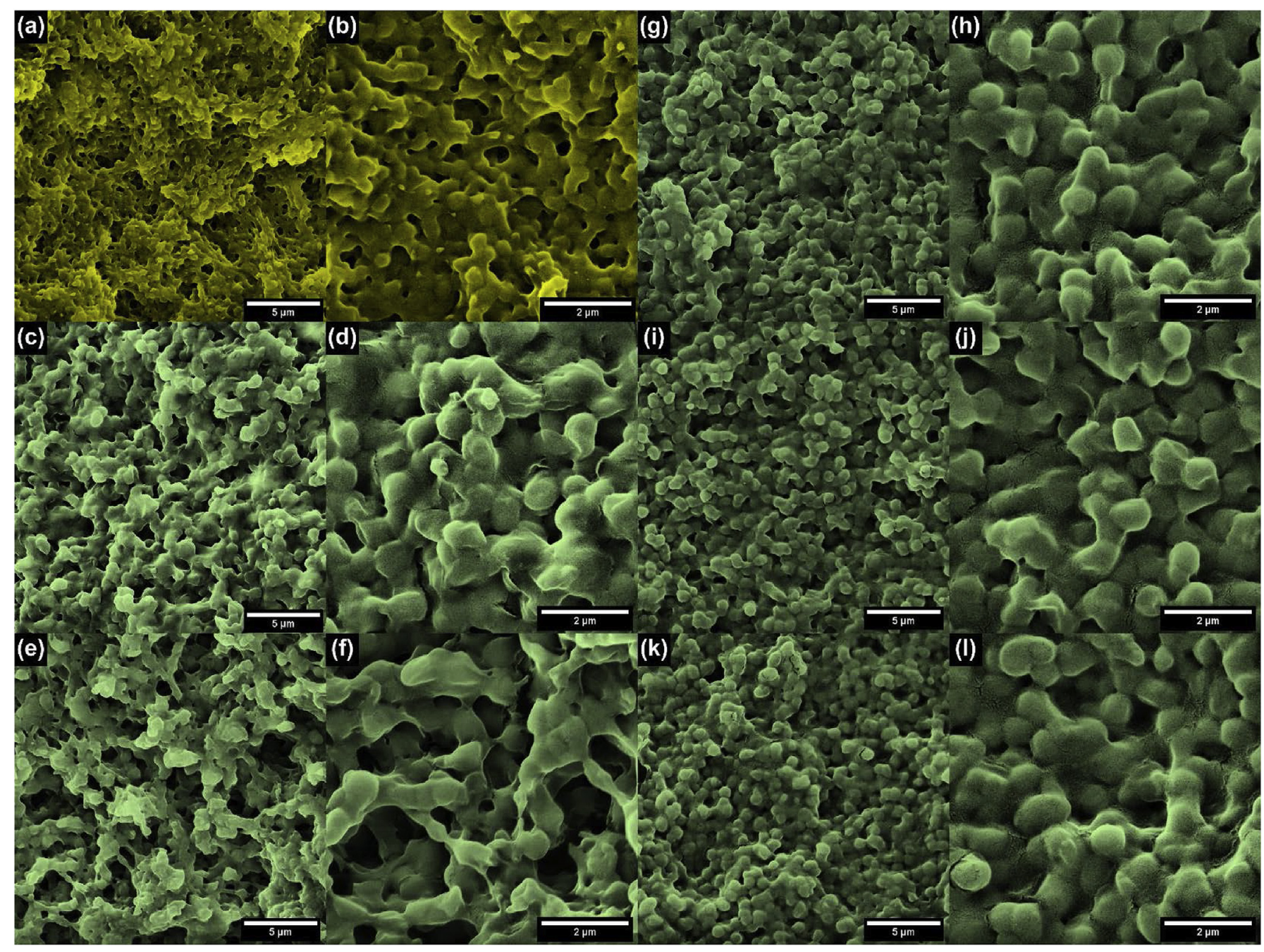

Fig. 4. SEM images of composite PLGA-PAA hydrogels: UV-PLGA-PAA (dose $3.6 \mathrm{~J} / \mathrm{cm}^{2}$; dose rate $1.2 \mathrm{~J} / \mathrm{cm}^{2} \mathrm{~min}$ ) ((a)-(b)) and G-PLGA-PAAs synthesized with different irradiation parameters (dose (kGy)/dose rate (kGy/h)): 17/8.1 ((c)-(d)), 25/6.1 ((e)-(f)), 25/8.1 ((g)-(h)), 25/10.1 ((i)-(j)), 33/8.1 ((k)-(l)).

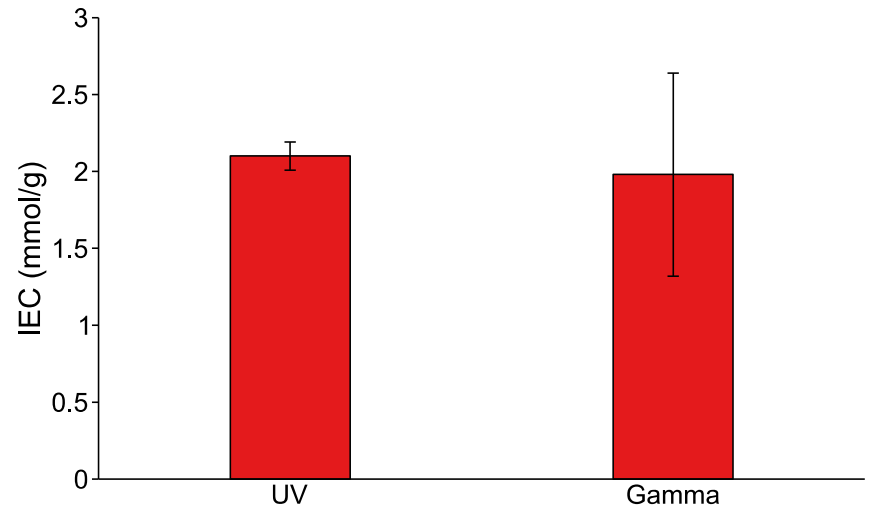

Fig. 5. Ion exchange capacity (IEC) of UV-PLGA-PAA and G-PLGA-PAAs.

by differences in irradiation parameters.

\subsection{Swelling kinetics}

Recorded swelling kinetics for composite PLGA-PAA hydrogels is shown in Fig. 7. Swelling kinetics of composite PLGA-PAA hydrogels approaches equilibrium after about $30 \mathrm{~min}$ for UV-PLGA-PAA (see Fig. 7a) and around $20 \mathrm{~min}$ for G-PLGA-PAAs (see Fig. $7 \mathrm{~b}$ and c). No lag is observed during swelling. The absence of swelling lag for all

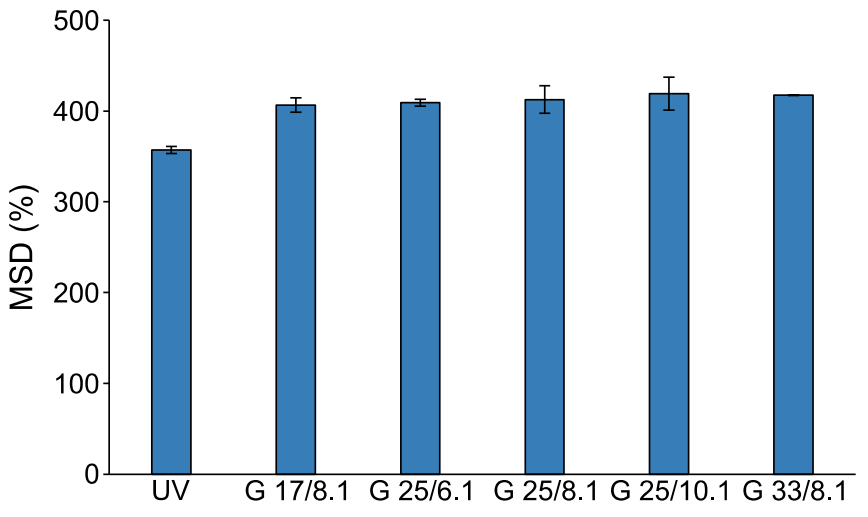

Fig. 6. Mass swelling degrees (MSDs) of UV-PLGA-PAA (dose $3.6 \mathrm{~J} / \mathrm{cm}^{2}$; dose rate $1.2 \mathrm{~J} / \mathrm{cm}^{2} \mathrm{~min}$ ) and G-PLGA-PAAs synthesized using different irradiation parameters (dose $(k G y) /$ dose rate $(k G y / h)$ ).

composite PLGA-PAA hydrogels can be explained by their porous nature in a dry state, which allows for the initial capillary action in addition to diffusion.

As in the case of equilibrium MSD, there is almost no difference in swelling dynamics among G-PLGA-PAAs. Swelling proceeds faster for G-PLGA-PAAs during the entire process. Faster swelling kinetics of GPLGA-PAAs can be mainly attributed to the presence of larger pores 

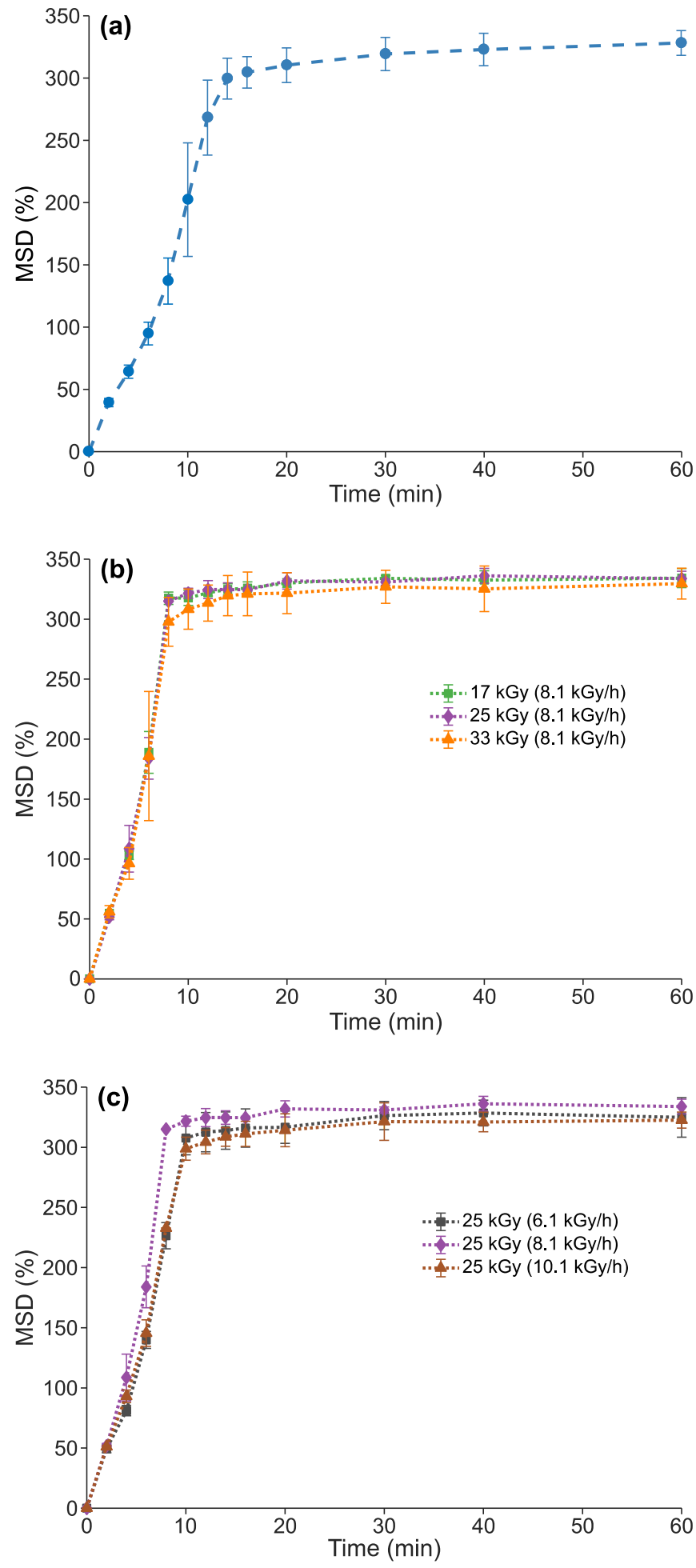

Fig. 7. Swelling kinetics of composite PLGA-PAA hydrogels: (a) UV-PLGA-PAA (dose $3.6 \mathrm{~J} / \mathrm{cm}^{2}$; dose rate $1.2 \mathrm{~J} / \mathrm{cm}^{2} \mathrm{~min}$ ), (b) G-PLGA-PAAs synthesized with the applied doses of 17,25 , and $33 \mathrm{kGy}$ (with the constant dose rate of $8.1 \mathrm{kGy} /$ h), and (c) G-PLGA-PAAs synthesized with the applied dose rates of 6.1, 8.1, and $10.1 \mathrm{kGy} / \mathrm{h}$ (with the constant dose of $25 \mathrm{kGy}$ ).

within the structure. G-PLGA-PAAs are also expected to be more hydrophilic due to the formation of additional polar carboxyl groups (Yoshioka et al., 1995).

MSD achieved after initial drying of composite PLGA-PAA hydrogels in the air at ambient conditions decreases for both types (approximately $8 \%$ for UV-PLGA-PAA and about $20 \%$ for G-PLGA-PAAs). Such findings indicate the lack of reversible swelling which is more pronounced for GPLGA-PAAs. The decrease in MSD can be attributed to the occurrence of pore collapse during drying, which is more probable for G-PLGA-PAAs due to the less homogeneous microstructure.

\section{Conclusions}

We have successfully synthesized composite PLGA-PAA hydrogels using a protocol which combines UV or gamma irradiation with the liquid phase inversion process. UV-PLGA-PAA and G-PLGA-PAAs were compared regarding microstructure, chemical composition, glass transition, swelling properties and capacity for storage of cationic drugs. Significant differences were observed between the two composite PLGA-PAA hydrogel types. Regarding gamma irradiation parameters, obtained material properties mainly depended on the absorbed dose, while no conclusive and systematic effects of dose rate were detected. All obtained composite PLGA-PAA hydrogels had a characteristic microstructure comprising porous PLGA-rich matrix with attached PAArich particle-like aggregates. G-PLGA-PAAs had a less uniform microstructure, larger pores, and greater PAA-rich aggregates. The chemical composition of UV-PLGA-PAA was quite homogeneous as opposed to the chemical composition of G-PLGA-PAAs. $\mathrm{T}_{g}$ of UV-PLGA-PAA increased, while the $T_{g}$ of G-PLGA-PAAs decreased in a dose-dependent manner. Swelling degree and swelling kinetics of G-PLGA-PAAs were improved compared to UV-PLGA-PAA and practically independent from the gamma irradiation parameters in the investigated range. None of the synthesized composite PLGA-PAA hydrogels were capable of fully reversible swelling. IEC of all composite PLGA-PAA hydrogels was quite similar. However, the IEC of G-PLGA-PAAs exhibited a significantly higher degree of variability.

UV-PLGA-PAA exhibits more reliable and robust material properties. G-PLGA-PAAs have some favorable swelling properties, their glass transition can be adjusted by changing the irradiation dose, and can be efficiently produced without the use of potentially hazardous PI. However, G-PLGA-PAAs suffer from structural and compositional inhomogeneities.

Our future research will be directed towards investigating the release of model cationic drugs from both types of composite PLGA-PAA hydrogels in vitro to evaluate the most important material properties which should be controlled and optimized. According to these results, further optimizations of gamma irradiation parameters and initial solution composition can be performed to obtain improved cationic drug reservoirs.

\section{Declarations of interest \\ None.}

\section{Acknowledgements}

The authors acknowledge Dr. Nenad Filipović from the Institute of Technical Sciences of the Serbian Academy of Sciences and Arts for assistance in thermal analysis. This work was supported by the Ministry of Education, Science and Technological Development of the Republic of Serbia (grant number TR32008).

\section{Appendix A. Supplementary data}

Supplementary data to this article can be found online at https:// doi.org/10.1016/j.radphyschem.2019.108466.

\section{References}

Abd El-Rehim, H.A., Hegazy, E.-S.A., Hamed, A.A., Swilem, A.E., 2013. Controlling the size and swellability of stimuli-responsive polyvinylpyrrolidone-poly(acrylic acid) nanogels synthesized by gamma radiation-induced template polymerization. Eur. 
Polym. J. 49, 601-612. https://doi.org/10.1016/j.eurpolymj.2012.12.002.

Brodbeck, K.J.J., Desnoyer, J.R.R., McHugh, A.J.J., 1999. Phase inversion dynamics of PLGA solutions related to drug delivery. Part II. The role of solution thermodynamics and bath-side mass transfer. J. Control. Release 62, 333-344. https://doi.org/10. 1016/S0168-3659(99)00159-5.

Callinan, T.D., 1956. Polymer synthesis by gamma radiation. J. Electrochem. Soc. 103, 292. https://doi.org/10.1149/1.2430313.

Carrascosa, C., Espejo, L., Torrado, S., Torrado, J.J., 2003. Effect of $\gamma$-sterilization process on PLGA microspheres loaded with insulin-like growth factor - I (IGF-I). J. Biomater. Appl. 18, 95-108. https://doi.org/10.1177/088532803038026.

Chu, C.C., Campbell, N.D., 1982. Scanning electron microscopic study of the hydrolytic degradation of poly(glycolic acid) suture. J. Biomed. Mater. Res. 16, 417-430. https://doi.org/10.1002/jbm.820160410.

Coqueret, X., 2008. Obtaining high performance polymeric materials by irradiation. In: Spotheim-Maurizot, M., Mostafavi, M., Douki, T., Belloni, J. (Eds.), Radiation Chemistry. EDP Sciences, France.

Dai, Z., Ronholm, J., Tian, Y., Sethi, B., Cao, X., 2016. Sterilization techniques for biodegradable scaffolds in tissue engineering applications. J. Tissue Eng. 7 204173141664881. https://doi.org/10.1177/2041731416648810.

Davison, L., Themistou, E., Buchanan, F., Cunningham, E., 2018. Low temperature gamma sterilization of a bioresorbable polymer. PLGA. Radiat. Phys. Chem. 143, 27-32. https://doi.org/10.1016/j.radphyschem.2017.09.009.

Ferry, M., Ngono-Ravache, Y., Aymes-Chodur, C., Clochard, M.C., Coqueret, X., Cortella, L., Pellizzi, E., Rouif, S., Esnouf, S., 2016. Ionizing radiation effects in polymers. Ref. Modul. Mater. Sci. Mater. Eng. https://doi.org/10.1016/B978-0-12-803581-8. 02095-6.

Gabbott, P., 2008. A practical introduction to differential scanning calorimetry. In Gabbott, P. (Ed.), Principles and Applications of Thermal Analysis. Blackwell Publishing Ltd, Oxford, UK, pp. 1-50. https://doi.org/10.1002/9780470697702.ch1.

Ghobashy, M.M., 2018. Ionizing radiation-induced polymerization. In: Djezzar, B. (Ed.), Ionizing Radiation Effects and Applications. InTech, pp. 113-134. https://doi.org/ 10.5772/intechopen.73234.

Graham, P.D., Brodbeck, K.J., McHugh, A.J., 1999. Phase inversion dynamics of PLGA solutions related to drug delivery. J. Control. Release 58, 233-245. https://doi.org/ 10.1016/S0168-3659(98)00158-8.

Gupta, B., Anjum, N., 2003. Plasma and radiation-induced graft modification of polymers for biomedical applications. In: Kausch, H., Anjum, N., Chevolot, Y., Gupta, B., Léonard, D., Mathieu, H.J., Pruitt, L.A., Ruiz-Taylor, L., Scholz, M. (Eds.), Radiation Effects on Polymers for Biological Use. Springer, Berlin, Heidelberg, pp. 35-61. https://doi.org/10.1007/3-540-45668-6_2.

Hasanain, F., Guenther, K., Mullett, W.M., Craven, E., 2014. Gamma sterilization of pharmaceuticals-A review of the irradiation of excipients, active pharmaceutical ingredients, and final drug product formulations. PDA J. Pharm. Sci. Technol. 68, 113-137. https://doi.org/10.5731/pdajpst.2014.00955.

Irgacure 819, 2006. Material Safety Data Sheet. Ciba Specialty Chemicals Corporation, Tarrytown, NY.

ISO/ASTM 51538, 2017. Practice for Use of the Ethanol-Chlorobenzene Dosimetry System. 2017.

Janićijević, Ž., Ninkov, M., Kataranovski, M., Radovanović, F., 2019. Poly (DL-Lactide-co- $\varepsilon$-Caprolactone)/Poly(Acrylic acid) composite implant for controlled delivery of cationic drugs. Macromol. Biosci. 19 (2), 1800322. https://doi.org/10. 1002/mabi.201800322.

Janićijević, Ž., Radovanović, F., 2018. Polyethersulfone/poly(acrylic acid) composite hydrogel membrane reservoirs for controlled delivery of cationic drug formulations. Polymer 147, 56-66. https://doi.org/10.1016/j.polymer.2018.05.065.

Jo, S.Y., Park, J.S., Gwon, H.J., Shin, Y.M., Khil, M.S., Nho, Y.C., Lim, Y.M., 2012. Degradation behavior of poly (l-lactide-co-glycolide) films through gamma-ray irradiation. Radiat. Phys. Chem. 81, 846-850. https://doi.org/10.1016/j.radphyschem. 2012.03.013.

Jouyban, A., Fakhree, M.A.A., Shayanfar, A., 2010. Review of pharmaceutical applications of N-methyl-2-pyrrolidone. J. Pharm. Pharm. Sci. 13, 524-535.

Keles, H., Naylor, A., Clegg, F., Sammon, C., 2014. Studying the release of hGH from gamma-irradiated PLGA microparticles using ATR-FTIR imaging. Vib. Spectrosc. 71, 76-84. https://doi.org/10.1016/j.vibspec.2014.01.012.

Keles, H., Naylor, A., Clegg, F., Sammon, C., 2015. Investigation of factors influencing the hydrolytic degradation of single PLGA microparticles. Polym. Degrad. Stab. 119, 228-241. https://doi.org/10.1016/j.polymdegradstab.2015.04.025.

Kim, S., Jeong, J.O., Lee, S., Park, J.S., Gwon, H.J., Jeong, S.I., Hardy, J.G., Lim, Y.M., Lee, J.Y., 2018. Effective gamma-ray sterilization and characterization of conductive polypyrrole biomaterials. Sci. Rep. 8, 1-10. https://doi.org/10.1038/s41598-01822066-6.

Kimura, H., Ogura, Y., 2001. Biodegradable polymers for ocular drug delivery. Ophthalmologica 215, 143-155. https://doi.org/10.1159/000050849.

Klose, D., Siepmann, F., Elkharraz, K., Krenzlin, S., 2006. How porosity and size affect the drug release mechanisms from PLGA-based microparticles. Int. J. Pharm. 314, 198-206. https://doi.org/10.1016/j.ijpharm.2005.07.031.

Kovács, A., Stenger, V., Fóldiák, G., 1987. Evaluation methods of the ethanol-monochlorobenzene dosimeter system. In: 6th Tihany Symposium on Radiation Chemistry. Hedvig, pp. 701-709.
Kumar, A., Pillai, J., 2018. Implantable drug delivery systems: an overview. In: Grumezescu, A.M. (Ed.), Nanostructures for the Engineering of Cells, Tissues and Organs: from Design to Applications. Elsevier Science, pp. 473-511. https://doi.org/ 10.1016/B978-0-12-813665-2.00013-2.

Lee, T.H., Wang, J., Wang, C.H., 2002. Double-walled microspheres for the sustained release of a highly water soluble drug: characterization and irradiation studies. J. Control. Release 83, 437-452. https://doi.org/10.1016/S0168-3659(02)00235-3.

Lee, J.S., Chae, G.S., Khang, G., Kim, M.S., Cho, S.H., Lee, H.B., 2003. The effect of gamma irradiation on PLGA and release behavior of BCNU from PLGA wafer. Macromol. Res. 11, 352-356. https://doi.org/10.1007/BF03218376.

Li, J., Mooney, D.J., 2016. Designing hydrogels for controlled drug delivery. Nat. Rev. Mater. 1, 1-18. https://doi.org/10.1038/natrevmats.2016.71.

Loo, J.S.C., Ooi, C.P., Boey, F.Y.C., 2005. Degradation of poly(lactide-co-glycolide) (PLGA) and poly(L-lactide) (PLLA) by electron beam radiation. Biomaterials 26 , 1359-1367. https://doi.org/10.1016/j.biomaterials.2004.05.001.

Makadia, H.K., Siegel, S.J., 2011. Poly Lactic-co-Glycolic Acid (PLGA) as biodegradable controlled drug delivery carrier. Polymers 3, 1377-1397. https://doi.org/10.3390/ polym 3031377.

Malinovskaja, K., Laaksonen, T., Kontturi, K., Hirvonen, J., 2013. Ion-exchange and iontophoresis-controlled delivery of apomorphine. Eur. J. Pharm. Biopharm. 83, 477-484. https://doi.org/10.1016/j.ejpb.2012.11.014.

Matusiak, M., Kadlubowski, S., Ulanski, P., 2018. Radiation-induced synthesis of poly (acrylic acid) nanogels. Radiat. Phys. Chem. 142, 125-129. https://doi.org/10.1016/ j.radphyschem.2017.01.037.

Montanari, L., Costantini, M., Signoretti, E.C., Valvo, L., Santucci, M., Bartolomei, M., Fattibene, P., Onori, S., Faucitano, A., Conti, B., Genta, I., 1998. Gamma irradiation effects on poly(DL-lactictide-co-glycolide) microspheres. J. Control. Release 56 , 219-229. https://doi.org/10.1016/S0168-3659(98)00082-0.

Mulder, M., 1996. Preparation techniques for immersion precipitation. In: Basic Principles of Membrane Technology. Kluwer Academic Publishers, Dordrecht, pp. $77-81$.

Okay, O., 2009. General properties of hydrogels. In: In: Gerlach, G., Arndt, K.-F. (Eds.), Hydrogel Sensors and Actuators. Springer Series on Chemical Sensors and Biosensors (Methods and Applications), vol. 6. Springer, Berlin, Heidelberg, pp. 1-14. https:// doi.org/10.1007/978-3-540-75645-3_1.

Oliveira, L.M., Araujo, P.L.B., Araujo, E.S., 2012. The effect of gamma radiation on mechanical properties of biodegradable polymers poly(3-hydroxybutyrate) and poly(3hydroxybutyrate-co-3-hydroxyvalerate). Mater. Res. 16, 195-203. https://doi.org/ 10.1590/S1516-14392012005000173.

Parent, M., Nouvel, C., Koerber, M., Sapin, A., Maincent, P., Boudier, A., 2013. PLGA in situ implants formed by phase inversion: critical physicochemical parameters to modulate drug release. J. Control. Release 172, 292-304. https://doi.org/10.1016/j. jconrel.2013.08.024.

Rafienia, M., Mirzadeh, H., Mobedi, H., Jamshidi, A., 2007. In vitro evaluation of drug solubility and gamma irradiation on the release of betamethasone under simulated in vivo conditions. J. Bioact. Compat Polym. 22, 443-459. https://doi.org/10.1177/ 0883911507080110

Rafienia, M., Emami, S.H., Mirzadeh, H., Mobedi, H., Karbasi, S., 2009. Influence of poly (lactide-co-glycolide) type and gamma irradiation on the betamethasone acetate release from the in situ forming systems. Curr. Drug Deliv. 6, 184-191. https://doi.org/ $10.2174 / 156720109787846243$.

Razem, D., Dvornik, I., 1972. Application of the ethanol-chlorobenzene dosimeter to electron-beam and gamma-radiation dosimetry: II Cobalt-60 grama rays. In: IAEA Symposium on Dosimetry in Agriculture, Industry, Biology, and Medicine, pp. 405-419 Vienna.

Schneider, C.A., Rasband, W.S., Eliceiri, K.W., 2012. NIH Image to ImageJ: 25 years of image analysis. Nat. Methods 9, 671-675. https://doi.org/10.1038/nmeth.2089.

Silva Aquino, K.A.da, 2012. Sterilization by gamma irradiation. In: Adrovic, F. (Ed.), Gamma Radiation. InTech, pp. 171-206. https://doi.org/10.5772/34901.

Sintzel, M.B., Merkli, A., Tabatabay, C., Gurny, R., 1997. Influence of irradiation sterilization on polymers used as drug carriers-a review. Drug Dev. Ind. Pharm. 23, 857-878. https://doi.org/10.3109/03639049709148693.

Sütekin, S.D., Güven, O., 2018. Radiation-induced controlled polymerization of acrylic acid by RAFT and RAFT-MADIX methods in protic solvents. Radiat. Phys. Chem. 142, 82-87. https://doi.org/10.1016/j.radphyschem.2017.01.046.

Todica, M., Stefan, R., Pop, C.V., Olar, L., 2015. IR and Raman investigation of some poly (acrylic) acid gels in aqueous and neutralized state. Acta Phys. Pol. A 128, 128-135. https://doi.org/10.12693/APhysPolA.128.128.

Vey, E., Rodger, C., Booth, J., Claybourn, M., Miller, A.F., Saiani, A., 2011. Degradation kinetics of poly(lactic-co-glycolic) acid block copolymer cast films in phosphate buffer solution as revealed by infrared and Raman spectroscopies. Polym. Degrad. Stab. 96, 1882-1889. https://doi.org/10.1016/j.polymdegradstab.2011.07.011.

Vuorio, M., Manzanares, J.A., Murtomäki, L., Hirvonen, J., Kankkunen, T., Kontturi, K., 2003. Ion-exchange fibers and drugs: a transient study. J. Control. Release 91, 439-448. https://doi.org/10.1016/S0168-3659(03)00270-0.

Yoshioka, S., Aso, Y., Otsuka, T., Kojima, S., 1995. The effect of $\gamma$-irradiation on drug release from poly(lactide) microspheres. Radiat. Phys. Chem. 46, 281-285. https:// doi.org/10.1016/0969-806X(95)00025-S. 